\title{
Génesis de una ciudad turística mexicana al comienzo del siglo XX: el antiguo centro minero de Guanajuato ${ }^{1}$
}

\author{
Oscar Sánchez Rangel* \\ Universidad de Guanajuato (México)
}

\begin{abstract}
Resumen: Durante la primera mitad del siglo XX, la ciudad de Guanajuato cambió su especialización como centro minero y entró en una redefinición para su consumo como espacio turístico. La viabilidad de la ciudad estuvo en riesgo por su escasa diversificación, el despoblamiento y la falta de comunicaciones. Sin embargo, la transformación de la minería, la reafirmación de Guanajuato como una ciudad administrativa y algunas iniciativas para el fomento del turismo contribuyeron a que esta ciudad delineara una nueva especialización económica. En este artículo proponemos una explicación acerca de la reconversión económica de la ciudad de Guanajuato, en que el turismo fue vital para la rehabilitación de sus espacios.
\end{abstract}

Palabras Clave: Turismo; Minería; Reconversión productiva; Emigración; Guanajuato.

Genesis of a Mexican tourist city at the beginning of the 20th century: the former mining center of Guanajuato

Abstract: During the first half of the 20th century, the city of Guanajuato changed its specialization as a mining center and entered into a redefinition for its consumption as a tourist space. The viability of the city was at risk due to its scarce diversification, depopulation and lack of communications. However, the transformation of mining, the reaffirmation of Guanajuato as an administrative city and some initiatives for the promotion of tourism contributed to this city delineate a new economic specialization. In this article we propose an explanation about the economic reconversion of the city of Guanajuato, where tourism was vital for the rehabilitation of its spaces.

Keywords: Tourism; Mining; Productive reorganization; Emigration; Guanajuato.

\section{Introducción}

En 1944, las estrellas cinematográficas Dolores del Río y Emilio "el Indio” Fernández buscaban locaciones para su próxima película, por lo que visitaron la capital del estado de Guanajuato, que consideraron idónea para sus planes debido a su carácter "netamente colonial". ${ }^{2} \mathrm{Al}$ año siguiente se estrenó Bugambilia, filme situado en el Guanajuato decimonónico, sobre el amor casi imposible entre la hija de un empresario minero y el capataz de la mina. Esta película, que inicia con una descripción idealizada de la ciudad, fue presentada como "el más claro exponente de las bellezas de la capital guanajuatense." ${ }^{3}$ Este pasaje es indicativo de la resignificación del antiguo centro minero de Guanajuato como un espacio de consumo, que le dio un nuevo sentido como patrimonio arquitectónico y cultural, al tiempo que se modificó la mirada del turista en torno a la ciudad, en tanto construcción social definida históricamente (Urry, 1990:1-3). El cambio de la ciudad de Guanajuato en un lugar turístico implicó la invención y revaloración de sus contenidos paisajísticos, así como de su historia, cultura y patrimonio. Se trata de un complejo proceso de mercantilización del espacio y de redefinición de las relaciones sociales que incluye dos dimensiones sustantivas. Por un lado, la apropiación, adaptación e invención de los sentidos culturales por parte de la industria, para redirigir la mirada sobre pueblos, ciudades, barrios o paisajes. La segunda dimensión consiste en el control del territorio y de los recursos estratégicos, que

Universidad de Guanajuato (México); E-mail: osrangel_@hotmail.com 
realizan distintos agentes para satisfacer la demanda de los visitantes, mediante el ejercicio del poder, con efectos sobre las relaciones sociales y materiales preexistentes (López y Marín, 2010: 222-229). Este artículo incorporará algunos aspectos de esas dos dimensiones, para analizar la reorganización de Guanajuato como un espacio turístico. No se pretende un enfoque integral del fenómeno, pues el énfasis recaerá en la redefinición productiva de la ciudad de Guanajuato durante las primeras décadas del siglo XX, para distinguir el tránsito de una economía basada en la minería hacia otra en que el turismo se convirtió en una actividad clave.

La ciudad de Guanajuato se había desarrollado desde el virreinato en torno a la extracción de plata, pero esta actividad perdió vitalidad en el curso de las primeras décadas del siglo XX, por lo que inclusive se pronosticó que se convertiría en un pueblo fantasma. ${ }^{4}$ Este riesgo se conjuró y actualmente el municipio de Guanajuato está habitado por más de 184 mil personas, cuya economía se fundamenta en la oferta de servicios, entre los cuales figura el turismo prominentemente. Guanajuato es uno de los diez estados del país con mayor afluencia de turistas al año y la capital se ubica como el segundo destino del estado, con más de medio millón de visitantes, solamente atrás de León, una de las ciudades más pobladas del país, que recibe por encima de un millón de visitantes anualmente, en gran medida por razones de trabajo (SECTUR, Guanajuato, 2012: 22-23). Las celebraciones del Festival Internacional Cervantino desde 1972, el nombramiento de la ciudad de Guanajuato como Patrimonio Cultural de la Humanidad, en 1988, y la realización del Guanajuato International Film Festival a partir de 1998, han influido en su proyección turística internacional.

Existen numerosos casos en el mundo de centros de producción y trabajo que cayeron en decadencia y abandono, pero que fueron revalorizados como lugares turísticos debido a su patrimonio arquitectónico y cultural, elementos que se afianzan como ventajas competitivas. Este fenómeno se ha presentado en antiguas zonas industriales, áreas portuarias, estaciones ferroviarias y zonas barriales de gran tradición, que se transformaron en espacios de consumo, en donde los servicios, el espectáculo, la explotación de la herencia cultural y la comercialización de la imagen predominan en su crecimiento económico y desarrollo social (López y Marín, 2010: 230). La reconversión de los centros mineros decadentes se complejiza, aunque se definan planes concretos, en función del grado de interdependencia entre la economía local y la minería, pues las alternativas quedan acotadas por la reducción y el envejecimiento poblacional, la baja inversión y la precariedad financiera del gobierno local (Cañizares, 2008: 13).

Desde una perspectiva más general, el tránsito hacia un nuevo tejido productivo depende de factores relativos a las condiciones históricas de las regiones y a las respuestas de los actores económicos, las organizaciones y las instituciones involucradas (Cerutti, 2008). Algunos estudios sobre el turismo en México durante el siglo XX muestran que esta actividad fue una opción en regiones cuyo tejido productivo tradicional declinó, pero la reconversión les abrió nuevas oportunidades. Así, en Yucatán y en el puerto sinaloense de Mazatlán, en donde la producción de henequén en el primer caso y de la minería y el comercio en el segundo, dejaron de funcionar como motor de las economías locales, el turismo delineó una ruta hacia la reconversión productiva, no exenta de dificultades, pero que los convirtió en destinos turísticos de primer orden en México (Anaya, 2015; Román y Aguilar, 2010). En este sentido, el propósito de nuestra investigación consiste en explicar el camino que siguió la ciudad de Guanajuato para transitar de una especialización minera hacia otra en torno a los servicios, en que el turismo fue el eje para la mercantilización del espacio.

Hasta hace poco predominaba la idea acerca de que el turismo en Guanajuato era resultado de acciones objetivas, como la creación del Departamento de Turismo del Estado, en 1961, o la apertura del primer hotel de cinco estrellas, en 1966 (Ayuntamiento de Guanajuato, 2009: 29). Esta interpretación despoja de complejidad al turismo, como proceso de mercantilización del espacio, que conjunta un reordenamiento económico, socioespacial, de las estructuras de poder y de transformación cultural. En esta dirección han avanzado los trabajos de Martínez (2009), Sánchez (2012) y Vidaurri (2014), quienes han encontrado evidencia de ese cambio histórico durante las primeras décadas del siglo XX, sobre todo en los años veinte. Esta reformulación en la periodización coincide con las investigaciones críticas de que la moderna industria turística mexicana haya sido resultado de las políticas públicas del gobierno de Miguel Alemán (1946-1952). En cambio, varios trabajos recientes explican el proceso a partir de las condiciones que condujeron a que el turismo se configurara como una experiencia internacional moderna (Berger, 2006; Berger y Grant, 2010: 6-7).

El turismo, como una manifestación en que el trabajo y el ocio se organizan separadamente en la sociedad, se integró en la vida moderna a lo largo de los siglos XIX y XX (Urry, 1990: 2, 18-19). El crecimiento del turismo durante el siglo XIX se debió a varios factores, como el cambio tecnológico en los transportes, con el uso del barco de vapor y del ferrocarril, el mejoramiento en las condiciones de 
salud, además de un conjunto de cambios sociales y culturales que alimentaron la curiosidad turística: la urbanización, las relaciones de género, la difusión de la arqueología, la historia y las ciencias en general, la invención de la fotografía, las prácticas deportivas, la accesibilidad a la música -particularmente a la ópera-, y el crecimiento de la industria editorial. En el siglo XX, la popularización del automóvil y más tarde del avión, hicieron accesibles más regiones y la formación de nuevas rutas turísticas. Las reformas laborales en diversos países que hicieron del tiempo libre un derecho de los trabajadores, con base en la reducción de la jornada laboral, el descanso dominical y las vacaciones pagadas, favorecieron al turismo como actividad comercial (Gordon, 2002: 126-133).

Una consecuencia adicional de la expansión del turismo en el mundo como actividad económica fue la intervención del Estado para su promoción, durante la primera mitad del siglo XX. (Gordon, 2002: 132 y 133). En México, el turismo se incorporó en los planes económicos gubernamentales desde finales de los años veinte, pues se asociaba con un capitalismo moderno y como una vinculación con el mundo, mediante la evocación de lo mexicano, entendido como un conjunto de expresiones nacionalistas. Esto implicaba la explotación comercial del paisaje natural, de los pueblos indígenas, del arte folklórico y de las construcciones monumentales, que incluía pirámides antiguas, edificios coloniales, monumentos porfirianos y construcciones modernas (Berger, 2006; Berger y Grant, 2010: 6-7).

Conviene considerar tres niveles de análisis complementarios para el estudio de la ciudad de Guanajuato como espacio turístico. Primero, el turismo como una industria global, que contribuye a la expansión capitalista, a la difusión de estilos de vida y del mercado mundial. Segundo, la influencia del Estado como rector, intermediario y regulador de los proyectos de modernización. Finalmente, la dimensión local, para identificar las dinámicas endógenas, los actores locales y las tendencias del mercado (López y Marín, 2010: 237). A lo largo de la exposición contemplaremos estas líneas de análisis para dilucidar los mecanismos de la transformación del tejido productivo en la ciudad de Guanajuato. Adicionalmente, sostenemos que la reconversión de esta ciudad fue parte de una ruptura histórica con respecto a la estructura económica de la región de estudio definida desde el virreinato, basada en la producción de plata en la sierra central de Guanajuato y de cereales básicos en el Bajío.

Para realizar esta investigación analizamos informes y correspondencia oficial procedente de archivos de Guanajuato, así como de diversos periódicos. El artículo incluye tres secciones, en la primera examinaremos el reajuste minero desde 1911, cuando la producción tendió a la baja y comenzaron a plantearse alternativas para la reactivación económica de la ciudad de Guanajuato, una de las cuáles fue el turismo. En la segunda sección propondremos que la reconversión económica fue obstaculizada por el despoblamiento y la falta de comunicaciones, aunque actuaron en sentido contrario la continuidad de la ciudad como capital estatal y centro administrativo. En la tercera sección examinaremos algunos elementos de la política de fomento y el surgimiento de organizaciones para la promoción del turismo, en las que colaboraron el gobierno y el sector privado.

\section{El turismo como derrota ante la crisis minera}

La reorganización de la ciudad de Guanajuato como espacio turístico fue uno de los cambios profundos en la economía y en la sociedad del estado de Guanajuato, durante las primeras décadas del siglo XX. La decadencia minera y el comportamiento errático de la producción de granos, principalmente de trigo y maíz, provocaron que Guanajuato se distanciara de la fisonomía económica prevaleciente desde el virreinato (Sánchez, 2012: 224-235). Al comienzo del siglo XX, la minería guanajuatense se reactivó mediante la inversión de capitales extranjeros, al mismo tiempo que aumentó la producción de trigo y maíz en el Bajío, que era la zona agrícola más fértil del estado y que le había dado fama como granero de Nueva España. Sin embargo, el panorama se enturbió, la minería y la agricultura decayeron y la emigración hacia Estados Unidos aumentó, de tal forma que, durante la década de 1920, el estado de Guanajuato se consolidó como un expulsor de población, junto con Jalisco y Michoacán (Gamio, 1930).

El centro minero de Guanajuato tuvo una fugaz bonanza al comienzo del siglo XX, en un momento en que, mediante inversiones extranjeras, la minería era la actividad más dinámica del sector exportador mexicano y pieza clave de la modernización emprendida durante el Porfiriato (1876-1911). Entre 1902 y 1907, Guanajuato recibió uno de los más elevados volúmenes de inversión en el país dirigidos hacia la minería, principalmente a través de empresas estadounidenses. La eficiencia en los procesos productivos aumentó debido al uso de electricidad y la sustitución del método de patio por el de cianuración, para la refinación de los minerales. La producción alcanzó un nivel máximo en 1911, pero posteriormente la tendencia cambió a la baja, por lo que a mediados del siglo XX se redujo en $90 \%$ respecto a la de 1911 
(Sánchez, 2015: 188-215). Esta contracción fue desastrosa para la ciudad, debido a la fuerte imbricación entre la minería y la vida local.

La orientación de la ciudad de Guanajuato como espacio turístico se vinculó con un ajuste, que contribuyó a que la minería no se contrajera todavía más. La decadencia minera concentró el mercado, pues cerraron empresas y hacia mediados del siglo XX solamente operaban tres, además de diversas explotaciones rudimentarias. ${ }^{5}$ Estas empresas redujeron sus inversiones, especialmente en la exploración de yacimientos, para concentrarse en el beneficio de los minerales con mayor contenido de plata y oro, pues el procesamiento de minerales pobres no era lucrativo. En las minas proliferaron buscones, mineros en pequeña escala a quienes los empresarios les pagaban un porcentaje de los minerales extraídos. También se expandieron los lupios, que explotaban minas abandonadas mediante métodos tradicionales, y a quienes frecuentemente se les acusaba de ladrones. Ambas formas de trabajo resurgían en cada crisis y se caracterizaron por una baja productividad. Otro cambio en la producción fueron las cooperativas de trabajadores de finales de la década de 1930, establecidas como respuesta a conflictos obrero-patronales. Estas cooperativas privilegiaron el empleo en lugar de la obtención de ganancias, lo que lograron parcialmente con la inversión de fondos públicos. El gambusinaje, la tolerancia hacia los lupios, así como las cooperativas contuvieron la reducción del empleo, aunque de cualquier forma cayó a un tercio entre 1900 y 1950 (Sánchez, 2012: 106).

Tomando en cuenta la magnitud del declive minero y la escasez de alternativas para la reactivación de la ciudad, el reajuste minero significó un piso, que atemperó la caída del empleo y le proporcionó un respiro a la trastocada economía local. Este precario piso contribuyó a la reconversión productiva pues, si el empleo minero hubiera caído más o se hubieran paralizado las actividades, como ocurrió durante la década de 1910, la reconversión se habría complicado más. Una mayor desocupación pudo tener consecuencias económicas más perjudiciales, debido a la contracción de la demanda comercial y de los ingresos para la tesorería local. La calidad de los servicios públicos se habría deteriorado en mayor medida, además de que el empobrecimiento de la población y la tensión social se habrían agravado.

¿La revaloración de la ciudad de Guanajuato como espacio turístico se vislumbró pronto como una alternativa al declive minero? ¿Qué opciones se plantearon? Como veremos, la idea de convertir a Guanajuato en una ciudad para el consumo de mercancías culturales y experiencias compitió con otras que insistieron en que debía preservarse la estructura económica tradicional, aunque también hubo propuestas para el fomento comercial e industrial. Además, hubo posturas contrarias al turismo, al igual que en otras partes del país. Cuando el gobierno federal perfiló al turismo como un instrumento para la recuperación económica tras la crisis internacional de 1929, algunos críticos opinaron que corrompería la identidad y cultura mexicanas. México podía convertirse en un "parque de diversiones hedonista" al servicio de los extranjeros, "otra Cuba", según creía el general José Quevedo, al frente del Departamento de Turismo en 1936 (Berger y Grant, 2010: 1).

En 1945, la Universidad de Guanajuato convocó a un concurso de propuestas para la revitalización de la economía local, pero solamente se presentaron un par de proyectos, uno de los cuáles insistía en la vocación minera de Guanajuato. En cambio, se aseguraba que los planes para convertirla en una ciudad turística equivalían a "un complejo de derrotismo". ${ }^{6}$ Este rechazo se había presentado anteriormente, por ejemplo, en un documento oficial de 1842 se afirmaba que la vocación económica de la ciudad no era "agrícola ni artista" sino que su principal giro "había sido y sería" la minería (Preciado, 2009: 36). En aquél momento se vislumbraba un repunte minero, lo que efectivamente ocurrió, pero tras el declive productivo que le siguió a la bonanza de principios del siglo XX, la insistencia en que la minería era la única vía para el desarrollo de Guanajuato era cada vez más irreal.

$\mathrm{Al}$ inicio del siglo XX casi toda la población de la ciudad de Guanajuato estaba vinculada al trabajo minero, por lo que el comercio dependía de los vaivenes en las minas. Había carnicerías, panaderías, talleres y establecimientos que ofrecían productos provenientes principalmente de la ciudad de México -abarrotes, vinos, ropa, ferretería, papelería, mercería, medicinas y artículos de lujo-, además de pequeños locales de servicios. La mayoría de los alimentos y del forraje provenía del Bajío: alfalfa, trigo, maíz, frijol, garbanzo y pajas de trigo y cebada. Había una pequeña producción industrial orientada al consumo local: tenerías, fábricas de hielo, jabón, chocolate, fideos, cigarros, aguas gaseosas y ladrillo. La alfarería alcanzó cierta notoriedad y se comercializó en mercados más lejanos. También destacó la explotación y labrado de canteras para la construcción y la fortificación de las minas (Sánchez, 2012: 112 y 113).

Esta escasa diversificación obstaculizó la viabilidad de la ciudad conforme avanzó la crisis minera, pues ofrecía pocas alternativas. Las propuestas para impulsar nuevas actividades productivas en la ciudad de Guanajuato proliferaron, sobre todo desde la década de 1920, cuando el ánimo de reconstrucción 
recorría al país después de los años más violentos de la Revolución mexicana. La rehabilitación de la minería fue una propuesta constante, pero también se mencionaron el comercio, la industria y el turismo como opciones para la reactivación económica. Algunos consideraban que la ciudad de Guanajuato podía convertirse en el principal centro de distribución comercial del estado debido a su localización geográfica, aunque otros plantearon que el camino a seguir era la industrialización. ${ }^{7}$

Una muestra de la sinuosa trayectoria que siguió la diversificación económica de la ciudad de Guanajuato es el trabajo de Francisco Antúnez Echagaray, como funcionario del gobierno del estado. Desde finales de la década de 1910, cuando apenas habían pasado los años más críticos de la Revolución mexicana y el centro minero de Guanajuato se había paralizado, Antúnez intentó convencer a diversos empresarios mineros para que invirtieran en el estado, pero sin éxito, pues la idea dominante era que los yacimientos estaban agotados. ${ }^{8}$ Tras este fracaso, Antúnez promovió la industrialización de la ciudad de Guanajuato y la construcción de carreteras para activar el comercio, además de que fue pionero en la promoción de éste como espacio turístico. En 1929, Francisco Antúnez redactó la ley sobre protección a la industria de Guanajuato, que otorgó facilidades fiscales para la fundación de nuevas industrias y, en cuanto a la ciudad, estableció franquicias para los empresarios, además de "apoyo moral y ayuda", aunque no se precisaba cómo se concretarían tales apoyos. ${ }^{9}$ Antúnez viajó a Estados Unidos para promover la nueva legislación, pero sin éxito. El cónsul honorario de México en Cleveland mostró poco interés en la industrialización de la ciudad de Guanajuato y, en cambio, propuso que se facilitaran las leyes locales de divorcio, para que los estadounidenses aprovecharan esa legislación, lo que impactaría positivamente en la economía local. Antúnez propuso a la compañía Amiesite Asphalt Co. of America que estableciera una planta de asfalto en la ciudad de Guanajuato, para aprovechar su localización en el centro del país, pero el plan no se concretó. ${ }^{10}$ Tras estos intentos fallidos, Antúnez participó en la definición de medidas para convertir a Guanajuato en un espacio turístico, como veremos más adelante.

Otras propuestas contemplaron la producción de alfarería que, como dijimos, gozaba de cierto prestigio, así como el establecimiento de fábricas de vidrio, porcelana, tubos de metal, material eléctrico, ropa y equipo militar - para aprovechar la proximidad de los campos militares en Irapuato, Sarabia, Guadalajara y Morelia- ${ }^{11}$ La actividad industrial creció marginalmente, pues solamente aparecieron pequeños establecimientos para la demanda local. ${ }^{12}$ Esta diversificación no debe desdeñarse, pues inyectó cierto dinamismo a la endeble economía de la ciudad, así como lo hizo la minería, con base en el reajuste productivo al que nos referimos antes. Por otro lado, entre los factores que explican este aletargado desempeño industrial destacan la escasez de comunicaciones entre la ciudad de Guanajuato y otras regiones, así como la competencia que ejercieron las ciudades del Bajío -Celaya, Irapuato y León-, que tenían una tradición manufacturera más arraigada y que resultaron más atractivas para los inversionistas. Así, mientras que diversos planes para la diversificación económica no prosperaron, solamente el desarrollo de la ciudad de Guanajuato como espacio turístico se fortaleció y significó su redefinición económica y cultural.

\section{Alcances y límites de la reconversión económica}

En 1949 el escritor Salvador Novo visitó la ciudad de Guanajuato para dirigir la puesta en escena de La danza macabra. En su crónica del viaje, Novo (1994: 273) describió la belleza de las calles y la majestuosidad del Teatro Juárez, pero agregó contundentemente: "Guanajuato debe de hallarse bien pobre." Novo relató que, mientras contemplaba el templo de Valenciana, salieron "seis o siete chiquillos conmovedoramente desnutridos y miserables" y que había encontrado "desoladoramente vacío" el mercado de dos pisos. La principal sorpresa del escritor fue que la ciudad lucía despoblada, "Llegó a tener seiscientos mil habitantes, y no le quedan más que veinte mil." Aunque la ciudad de Guanajuato jamás concentró seiscientos mil habitantes, la exageración de Salvador Novo refleja la percepción acerca de la severa reducción demográfica que había ocurrido. La caída poblacional fue uno de los costos más visibles de la reconversión productiva, como resultado del comportamiento de la minería, su efecto en el empleo minero y su relación con otras actividades, en las condiciones de la pobre diversificación económica local.

La crisis minera y su impacto sobre los negocios locales provocaron que muchas familias que dependían del empleo minero abandonaran la ciudad, de tal forma que la población en el municipio pasó de casi 67 mil habitantes en 1910 a casi 33 mil en 1921. Lo más notable fue la lenta recuperación, ya que el número de habitantes de 1910 apenas se recuperó durante la década de 1970, lo que evidencia las enormes dificultades que afectaron la reconfiguración de la ciudad tras la pérdida de la centralidad histórica de 
la minería. La emigración fue una opción creciente, al grado que la estructura poblacional por edades revela que el municipio de Guanajuato registró la mayor reducción en el intervalo de 20 a 39 años entre 1930 y 1950, lo que indica la expulsión de trabajadores (Sánchez 2012: 140 y 261). Esto favoreció el envejecimiento de la población, una variable que limitó la diversificación de las actividades. Este fenómeno es recurrente en las regiones mineras decadentes, pues la desocupación induce la emigración y las tasas de natalidad decrecen, lo que explica el envejecimiento progresivo de los habitantes (Romero y Santiago, 2010: 584). La estructura demográfica resultante puede obstaculizar el desarrollo de nuevas actividades debido a la falta de trabajadores, sobre todo de los especializados, como ha ocurrido en diversos centros mineros en declive que se reconvierten en lugares turísticos (Valenzuela, Palacios e Hidalgo, 2008: 242).

Un factor más que obstaculizó al incipiente turismo en Guanajuato consistió en la violencia derivada del enfrentamiento entre el Estado y la Iglesia, que condujo a la guerra cristera (1926-1929), y que generó una tensión que prevaleció hasta 1938. Esta confrontación tuvo como principal teatro de operaciones varios estados del centro occidente de México, sobre todo Jalisco, Guanajuato y Michoacán. Algunos estudiosos estiman que la violencia de estos años fue mayor que durante la Revolución mexicana, además de que agudizó la emigración, que había aumentado desde los años veinte en los estados mencionados (Gamio, 1930: 66 y 67; Durand, 1991: 10). El monumento a Cristo Rey ubicado en Silao, a unos minutos de la ciudad de Guanajuato, que actualmente es visitado por creyentes católicos y turistas, fue destruido por la aviación del ejército federal, lo que atizó los levantamientos. Estas condiciones afectaron al turismo, pues la paz y la estabilidad son clave para el éxito de esta industria. (Berger y Grant, 2010: 7). Aunque la violencia en Guanajuato se concentró al norte del estado, probablemente las noticias y rumores acerca de los conflictos, además del bandidaje asociado con esta clase de problemas, inhibieron a muchos de los turistas potenciales.

Mientras que la demografía y la violencia afectaron el desarrollo del turismo, en sentido opuesto actuó la permanencia de la ciudad de Guanajuato como capital estatal y, por lo tanto, sede de los poderes políticos locales. De esta manera, Guanajuato reafirmó su condición como una ciudad administrativa, de tal manera que su reordenamiento como ciudad turística constituyó un paso adicional hacia una especialización basada en la oferta de servicios. El riesgo de que Guanajuato perdiera su estatus como capital del estado era real, debido a la rivalidad histórica con la vecina ciudad de León, localizada en el corazón del Bajío. En 1915, en plena Revolución mexicana, las fuerzas de Pancho Villa nombraron a León como capital del estado, pero tras unos meses la medida se revirtió, aunque los comerciantes e industriales leoneses insistieron en que León debía de ser la capital estatal (Blanco, Parra y Ruíz, 2000: 178). La constitución local, promulgada en 1917, incluyó candados jurídicos para que se dificultara un posible cambio de la capital, con el propósito de que contribuyera a la alicaída economía de la ciudad de Guanajuato (Sánchez, 2012: 125). Esto garantizó la continuidad de la ciudad como centro administrativo, favoreció la aglomeración de las oficinas públicas y del personal respectivo, además de que alentó un flujo de población flotante. En 1945 fue construido un edificio sobre las ruinas de la antigua Casa de Moneda, que concentró las oficinas federales de correos, telégrafos y hacienda. ${ }^{13}$ Este perfil administrativo se fortaleció con el Colegio del Estado, que alimentaba el prestigio cultural que históricamente conservó la ciudad de Guanajuato. La afirmación de que Guanajuato era una "ciudad culta" fue utilizada frecuentemente para diferenciarse de León. Durante la primera mitad del siglo XX el Colegio acrecentó sus instalaciones, la comunidad académica se volvió más numerosa y en 1945 se transformó en la Universidad de Guanajuato (Rábago, 1997: 97-144). En 1925 se inscribieron cien estudiantes, pero en 1947 el número creció a 1,256, de los cuales 60 por ciento estudiaba en la ciudad de Guanajuato y el resto en León y Celaya. Para 1951 la población estudiantil había crecido en 70 por ciento al registrarse más de 2,100 alumnos (Guerrero, 1991: 707). Es así que, hacia mediados del siglo $\mathrm{XX}$, era evidente la conformación de un tejido productivo con un predominio de los servicios pues, de acuerdo con un informe del gobierno del estado, la economía de la ciudad de Guanajuato se sustentaba en cuatro elementos: la burocracia, el turismo, la Universidad y, en último lugar, la minería. ${ }^{14}$ De esta manera, la histórica ciudad minera era cosa del pasado.

Un cuello de botella en el desarrollo de infraestructura turística fue la falta de comunicaciones. Una nota periodística de 1928 cuestionaba la condición de Guanajuato como capital estatal, pues solamente contaba con "una estación terminal de vigésimo orden, y una mala carretera que llega hasta Silao", lo que dificultaba el contacto con las distintas poblaciones. ${ }^{15}$ Esta situación contrasta con la de Taxco, otro famoso centro minero localizado en Guerrero, que declinó a principios del siglo XX. La carretera México-Acapulco, inaugurada en 1928, fue vital para el resurgimiento de Taxco, que se convirtió en sede de talleres de joyería de plata, residencia de numerosos artistas y atractivo para turistas nacionales y 
extranjeros. Este reordenamiento económico, socioespacial y cultural provocó un aumento poblacional de 2,371 a 10 mil habitantes entre 1921 y 1950, un ritmo de crecimiento contrario al aletargamiento demográfico de la ciudad de Guanajuato (Babini, 2005: 95; Leal, 2007: 116). Durante la década de 1920, el principal proyecto vial en torno a la ciudad de Guanajuato consistió en la construcción de la carretera a Dolores Hidalgo, que se anunció como la clave para el resurgimiento de la ciudad, que contribuiría a que "sea la capital de grandezas que fuera en otros tiempos." ${ }^{16}$ La idea era facilitar las comunicaciones con el norte del estado, para la ampliación del abastecimiento de alimentos de la ciudad y también para favorecer el turismo, debido a que se consideraba que el paisaje natural del trayecto, "excepcionalmente pintoresco", era un atractivo adicional. ${ }^{17}$ Con el tiempo, la infraestructura de comunicaciones se expandió más en las ciudades del Bajío que en cualquier otra región del estado, de tal manera que la ciudad de Guanajuato reforzó su vinculación histórica con esas ciudades, aunque en una posición subsidiaria debido a su declive económico.

\section{La revaloración de la ciudad de Guanajuato como lugar turístico}

A finales de la década de 1920 el arribo de excursiones turísticas a Guanajuato era cada vez más frecuente, pero aún causaba sorpresa entre la población. La prensa local informaba sobre la llegada de visitantes extranjeros en un tono que confirma lo novedoso del fenómeno. En 1926, en una crónica periodística sobre el arribo de turistas extranjeros se leía: "Por la noche, como a las 24 horas, el tren especial que conduce a estos viajeros, se alejó de la Estación de Tepetapa, ante la presencia de numeroso público que a esa hora aun paseaba por los andenes de la estación." Otro diario informaba que "en el transcurso de la semana que hoy empieza visitarán a Guanajuato gran número de excursionistas." ${ }^{18}$ En 1931, cuando la minería sufría el impacto de la depresión internacional, el gobernador Agustín Arroyo (1991: 389) informó que Guanajuato había recibido excursiones de turistas estadounidenses. Esta fue la primera mención sobre el turismo en un informe del gobierno del estado.

La mercantilización para el consumo de la ciudad de Guanajuato como lugar turístico incluyó la formación de organizaciones y la implementación de medidas de fomento, en concordancia con los planes del gobierno federal, lo que implicó distintos niveles de coordinación con el gobierno estatal. A finales de la década de 1920 el gobierno federal reconoció formalmente los beneficios económicos del turismo y comenzó con el estudio, la organización y la promoción de esta actividad. En 1928, el gobierno federal creó la Comisión Pro-Turismo, dependiente de la Secretaría de Gobernación, dedicada a estudiar y recomendar acciones para el desarrollo turístico. Poco después surgió la Comisión Mixta Pro-Turismo, que incorporó a representantes del sector privado. En la coyuntura de la crisis internacional de 1929 el turismo adquirió una importancia especial para la política económica mexicana, porque atraía divisas necesarias para la reducción del déficit en la balanza de pagos (Berger, 2006: 2-7). El turismo extranjero creció rápidamente durante la década de 1930, alentado por la devaluación monetaria y la construcción de carreteras, por lo que las divisas obtenidas equivalieron al 7\% de las exportaciones de mercancías en 1940 (Cárdenas, 1987: 42).

Las comisiones pro-turismo también surgieron en Aguascalientes, Baja California, Coahuila, Colima, Guadalajara, Michoacán, Querétaro, San Luis Potosí, Tabasco y Yucatán. En 1929, por acuerdo del gobierno de Guanajuato, se fundó la Comisión Pro-Turismo de la ciudad de Guanajuato, cuyo primer presidente fue Francisco Antúnez Echagaray. ${ }^{19}$ Como dijimos, Echagaray intentó fallidamente atraer inversiones hacia la minería y para la industrialización de la ciudad, pero a finales de los años veinte se convirtió en un promotor del turismo. Esta Comisión se encargó de la promoción turística de Guanajuato en México y en el extranjero, sobre todo en Estados Unidos, así como del estudio de medidas para el fomento de esta actividad. La atracción de turistas estadounidenses fue prioritaria, por lo que a principios de la década de 1930 el gobierno del estado y algunos empresarios editaron un folleto en inglés titulado Guanajuato. Pictorial México. ${ }^{20}$ La carátula estaba ilustrada con una fotografía panorámica de la ciudad de Guanajuato y al reverso se encontraba un listado de las empresas que colaboraron en la publicación: Guanajuato Consolidated Mining and Milling Co., De Votie and Lujan S.A., Cámara Nacional e Industrial de León, Banco de Londres y México, S.A. (León), Banco Nacional de México (Irapuato), Compañía Hidroeléctrica Guanajuatense, S.A. y Mexican Telephone and Telegraph Company. Asimismo, en 1934, el gobierno de Guanajuato nombró a un representante en Nueva Orleans, con el fin de atraer a los turistas estadounidenses. ${ }^{21}$ Por otro lado, en 1939 fue instalada una oficina en la ciudad de México para la promoción del turismo en Guanajuato. ${ }^{22}$ En cuanto a la legislación, destaca la Ley de planeación del Estado de Guanajuato, que incluyó disposiciones para la planeación urbanística, 
así como la formación de una comisión que vigilaría la conservación de los monumentos artísticos e históricos y que funcionaría como órgano consultivo para la construcción de obras públicas en los municipios y ciudades. ${ }^{23}$ Además, en 1942, el congreso local declaró a la ciudad de Guanajuato "Ciudad histórica y típica”. Esta denominación se fundamentó en una ley que normó el crecimiento urbano y la conservación de los inmuebles de la ciudad, para preservar "su aspecto de población antigua" ${ }^{24}$ un rasgo de la transformación del patrimonio arquitectónico para el consumo.

En la oferta turística mexicana, la ciudad de Guanajuato se colocó como un espacio asociado con la noción del "México viejo", debido a su patrimonio arquitectónico de origen colonial, lo mismo que ciudades como México, Monterrey, Puebla y Taxco. De acuerdo con Alex Saragoza (2001: 96-100) esta vertiente turística, que implicaba la construcción y restauración de museos y monumentos para la exhibición del patrimonio nacional, fue de las principales influencias que modelaron la oferta turística mexicana de aquéllos años, que se definía en el nivel local y constituía un vínculo con la política federal de promoción turística. Otras influencias fueron el indigenismo, relacionado con los sitios arqueológicos, la recuperación y representación del México "auténtico", asociado con el concepto de folklore y la recuperación de la cultura vernácula. Este tipo de espacios turísticos, orientados al consumo de las ciudades como poblados típicos de origen colonial, también se desarrolló en otro municipio guanajuatense, San Miguel de Allende, localizado en la sierra central del estado, lo mismo que la ciudad de Guanajuato. La conversión de San Miguel de Allende en un espacio turístico, le permitió contrarrestar el decrecimiento de sus actividades tradicionales, -agricultura, ganadería y manufactura de textiles-, afectadas por la violencia de la guerra cristera, la reforma agraria y la emigración. El gobierno y distintos individuos y agrupaciones privadas construyeron gradualmente la identidad turística de San Miguel como un "típico pueblo mexicano", debido a su arquitectura colonial y porque formó parte del teatro de operaciones en el curso de la guerra de independencia, lo mismo que la ciudad de Guanajuato. El establecimiento de escuelas de arte, el arribo de intelectuales extranjeros bohemios y de veteranos del ejército estadounidense le imprimieron fuerza al turismo en San Miguel de Allende, que hacia 1950 era reconocida internacionalmente (Pinley, 2010).

Un resultado inicial de las acciones para el fomento turístico de la ciudad de Guanajuato fue la formación de una heterogénea agenda de trabajo: desarrollo de vías de comunicación, reglamentación de servicios turísticos, medidas para la conservación, limpieza y construcción de infraestructura, suministro de agua potable, establecimiento de una policía eficiente, dotación de servicios turísticos, como guías y centros de información, así como una política para el cuidado del patrimonio arquitectónico de la ciudad. ${ }^{25}$ La pobreza de la tesorería municipal limitó las acciones, un problema común en las regiones mineras en declive, que dificulta la reconversión económica en la medida en que la calidad de los servicios públicos se deteriora y no es posible construir la infraestructura pública necesaria para el desarrollo de nuevas actividades (Valenzuela, et al., 2008: 242). Al respecto, en la prensa se publicaron críticas hacia los organismos encargados del fomento turístico, acusaciones a los funcionarios por "apáticos", así como denuncias por el deterioro de los monumentos arquitectónicos. En 1936, un diario denunció el abandono del patrimonio artístico colonial, debido al estado lamentable del templo de San Juan de Rayas "totalmente desmantelado, ruinoso y en vías de desaparecer", así como el de Valenciana "que está por desplomarse y anchas grietas dolorosas abren sus muros". En 1944, el rector de la Universidad Nacional visitó Guanajuato y afirmó que era una ciudad turística de primer orden, pero lamentó que la Alhóndiga de Granaditas estaba convertida en "una pocilga inmunda"26 En 1949, en otro diario se denunció el mal estado del famoso Callejón del Beso, por lo que se urgía a los miembros del Comité Pro-Turismo a atender el asunto "antes que pedir a gritos que vengan centenares de visitantes a ver lo mal organizados que estamos". ${ }^{27}$

Estas críticas eran frecuentes en otros lugares turísticos de México pues, como ha afirmado Dina Berger (2006: 7), esta etapa se caracterizó por la pobre organización, las bajas ganancias, el pequeño flujo de visitantes y la mala prensa, aunque las cosas mejoraron a partir de la década de 1930. A pesar de las deficiencias y las limitaciones presupuestales, la ciudad de Guanajuato transitó de manera incipiente hacia una nueva especialización económica. Este proceso incluyó acciones objetivas, como la construcción y mejoramiento de la infraestructura urbana, que se realizó cada vez más explícitamente con el propósito de redefinir a la ciudad como un espacio turístico. ${ }^{28} \mathrm{La}$ falta de agua fue una restricción mayúscula para el turismo; el escritor Salvador Novo (1994: 272) recuerda que visitó Guanajuato en 1949 y el agua del hotel le parecía orines, "un agua turbia y amarilla que no debe beberse". ${ }^{29} \mathrm{~A}$ mediados del siglo XX se implementó una solución parcial para este problema, mediante la construcción de algunas obras hidráulicas. En 1949 comenzó la construcción de la Presa de la Soledad y en 1955 fue instalada la primera planta de tratamiento en el Mineral de Valenciana. Las obras de ornato también fueron realizadas con propósitos turísticos, como la construcción del monumento al Pípila (1939), así como la 
restauración del patrimonio histórico de la cuidad, por ejemplo, la remoción del palacio del Conde de Valenciana (1945), el reacondicionamiento de la Alhóndiga de Granaditas (1949), que había funcionado como cárcel, o la remodelación de la Universidad a finales de la década de 1940, que con el tiempo se convirtió en un atractivo turístico.

A mediados del siglo XX, Guanajuato estaba incluido en la oferta turística mexicana, que se concentraba en algunos estados. Una estadística indica que Guanajuato fue la sexta entidad por el número de establecimientos de hospedaje y la séptima de acuerdo con la cantidad de cuartos de hospedaje en México (Álvarez, 1960: 300-301). La oferta turística del estado estaba distribuida entre sus principales ciudades, que formaban uno de los sistemas urbanos más importantes de México. Pocos estados del país contaban con el número de ciudades que existían en el Bajío, lo que se presentó como un atractivo para los visitantes.. La ruta turística Lagos-Ciudad de México, que pasaba por doce ciudades en cuatro estados del país, incluía siete ciudades guanajuatenses: San Miguel de Allende, Celaya, Salamanca, Irapuato, Guanajuato, Silao y León. Aunque la ciudad de Guanajuato perdió la importancia poblacional que había tenido en el curso del siglo XIX, el hecho de que formara parte de esta concentración urbana favoreció su redefinición como lugar turístico en el siglo XX. La terciarización económica de la ciudad se consolidó durante el resto del siglo, con el turismo como actividad central, mientras que la minería ratificó su carácter secundario en la economía local.

\section{Conclusiones}

Durante las primeras décadas del siglo XX, en la ciudad de Guanajuato se desarrolló un proceso que la convirtió gradualmente en un espacio para su consumo turístico, lo que implicó cambios en su especialización productiva, de reorganización del espacio y de resignificación de su patrimonio arquitectónico y cultural. Esta mercantilización del espacio fue parte de una transformación de dimensiones internacionales, que consistió en la reestructuración de distintos lugares para su consumo turístico, implicó una creciente importancia económica del turismo, así como una mayor influencia de sus repercusiones sociales. La consolidación del turismo en la sociedad moderna coincidió con el declive minero de la ciudad de Guanajuato, lo que contribuyó a su supervivencia mediante una profunda redefinición económica. Conviene subrayar la magnitud del cambio, pues la minería fue el motor económico de la ciudad de Guanajuato desde el virreinato y había sido fundamental para su articulación social y cultural.

En cuanto a la nueva especialización productiva, debe destacarse la importancia del reajuste minero, pues proporcionó una base mínima, que formó parte de la ruta que siguió Guanajuato para convertirse en una ciudad turística. Este reajuste no detuvo la caída de la producción ni del empleo en la minería, pero implicó un nivel de actividad que absorbió una porción de la demanda laboral y le dio cierto soporte al comercio local. De cualquier forma, el empleo minero tendió a la baja, lo que afectó el comportamiento poblacional, pues la emigración fue una constante, de tal forma que el cambio hacia una economía de servicios estuvo condicionada por una oferta laboral escasa y envejecida, que se revirtió con mucha lentitud. La permanencia de Guanajuato como capital estatal y sede de la Universidad de Guanajuato, contuvieron la emigración, alentó los flujos de población flotante y contribuyeron a que Guanajuato se perfilara como una ciudad administrativa, es decir, estructurada en torno a la prestación de servicios. Las minas se mantuvieron con altibajos durante el resto del siglo XX, pero quedó atrás su papel central en la economía citadina. La génesis del nuevo tejido productivo, con la construcción de esta ciudad como un lugar turístico, y el acento en la producción de servicios, fue una faceta del cambio histórico en la región que comprende el estado de Guanajuato, que rompió con la fisonomía económica definida durante el virreinato. La transformación de la ciudad de Guanajuato fue lenta y tuvo consecuencias severas para una parte amplia de la sociedad, prueba de ello fue su comportamiento demográfico y la pérdida de su envergadura en la jerarquía urbana nacional, pero la resignificación de su patrimonio histórico y cultural construyó un horizonte con mayores posibilidades, que la especialización minera no ofreció.

\section{Archivos}

AHGEG, SG-TD: Archivo Histórico General del Gobierno del Estado de Guanajuato. Fondo Secretaría de Gobierno. Sección Tercer Departamento.

AHUG, CPA: Archivo Histórico de la Universidad de Guanajuato, Colección Ponciano Aguilar. 


\section{Hemerografía}

EN: El Noticioso, Guanajuato, Gto.

EG: Estado de Guanajuato, Guanajuato, Gto.

GDB: Guanajuato. Diario del Bajio, Irapuato, Gto.

POEG: Periódico Oficial del Estado de Guanajuato, Guanajuato, Gto.

\section{Bibliografía}

Álvarez, José

1960. "El Turismo", en México. Cincuenta años de Revolución. Tomo I. La Economía (pp. 291-309).

México: Fondo de Cultura Económica.

Anaya Merchant, Luis

2015. "Henequén, chicle y turismo. el financiamiento estatal para la transformación de la economía yucateca, 1915-1970”. En Legajos, número 7, julio-septiembre: pp. 35-59.

Arroyo, Agustín

1991. "Informe que rindió Agustín Arroyo Ch., el 15 de septiembre de 1931". En Guanajuato en la voz de sus gobernadores. Compilación de Informes de Gobierno 1917-1991, tomo 1 (pp. 375-406). Guanajuato, México: Gobierno del Estado de Guanajuato.

Ayuntamiento de Guanajuato

2009. Plan de gobierno municipal de Guanajuato 2009-2012. Guanajuato, México: Ayuntamiento de Guanajuato.

Babini Baan, Andrea

2005. Taxco de Alarcón. Evolución de una antigua ciudad minera. Chilpancingo, Guerrero: Universidad Autónoma de Guerrero.

Berger, Dina

2006. The development of México's Tourism Industry. Piramids by day, martinis by Night. United States of America: Palgrave Macmillan.

Berger, Dina y Andrew Grant

2010. "Introduction: tourism studies and the tourism dilemma". En Dina Berger y Andrew Grant (eds.), Holiday in Mexico. Critical reflections on tourism and tourist encounters (pp. 1-20). United States of America: Duke University Press, Durham and London.

Blanco, Mónica, Alma Parra y Ethelia Ruiz

2000. Breve historia de Guanajuato. México: Fondo de Cultura Económica.

Cañizares Ruiz, María del Carmen

2008. "El atractivo turístico de una de las minas de mercurio más importantes del mundo: el parque minero de Almadén (Ciudad Real). En Cuadernos de Turismo, Universidad de Murcia, España, no. 21: pp. 9-31.

Cárdenas Sánchez, Enrique

1987. La industrialización mexicana durante la Gran Depresión. México: El Colegio de México.

Cerutti, Mario

2008. "Crisis y reconversión del tejido productivo en un espacio regional del norte de México: La Laguna (1875-1975)”. En Investigaciones de Historia Económica, invierno, número 10: pp. 97-128.

Durand, Jorge

1991. "Presentación”, en Jorge Durand, (comp.), Migración México-Estados Unidos. Años veinte (pp. 9-17). México: Consejo Nacional para la Cultura y las Artes.

Gamio, Manuel

1930. Mexican inmigration to the United States. A study of human migration and adjustment. Chicago, Illinois: The University of Chicago Press,.

Gordon, Bertram

2001. "El turismo de masas: un concepto problemático en la historia del siglo XX". En Historia contemporánea, número 25: pp. 125-156.

Guerrero, Nicéforo

1991. "Informe que rindió Nicéforo Guerrero, el 15 de septiembre de 1947". En Guanajuato en la voz de sus gobernadores. Compilación de Informes de Gobierno 1917-1991, tomo 1 (pp. 693-744). Guanajuato, México: Gobierno del Estado de Guanajuato. 
Leal, Felipe

2007. "Taxco y la carretera a Acapulco". En Fernando Curiel (ed.), Taxco. La perspectiva urbana (pp. 111-124). México: Universidad Nacional Autónoma de México.

López Santillán, Ángeles y Gustavo Marín Guardado

2010. "Turismo, capitalismo y producción de lo exótico: una perspectiva crítica para el estudio de la mercantilización del espacio y la cultura". En Relaciones. Estudios de historia y sociedad, vol. XXXI, núm. 123, El Colegio de Michoacán: pp. 219-258.

Martínez Ortega, Claudia Janet

2009. La formación turística de la ciudad de Guanajuato. México, Guanajuato: Universidad de Guanajuato, División de Ciencias Sociales y Humanidades, Departamento de Historia, Tesis de Licenciatura en Historia.

Novo, Salvador

1994. La vida en México en el período presidencial de Miguel Alemán, compilación y nota preliminar de José Emilio Pacheco, Colección Memorias Mexicanas. México: Instituto Nacional de Antropología e Historia, Consejo Nacional para la Cultura y las Artes.

Pinley Covert, Lisa

2010. "Colonial outpost to artist's mecca: conflicto and collaboration in the development of San Miguel de Allende's tourist industry". En Dina Berger y Andrew Grant (eds.), Holiday in Mexico. Critical reflections on tourism and tourist encounters (pp. 183-220). United States of America: Duke University Press, Durham and London.

Preciado de Alba, Carlos Armando

2007. Guanajuato en tiempos de la intervención francesa y el Segundo Imperio. Guanajuato, México: Universidad de Guanajuato, Centro de Investigaciones Humanísticas.

Rábago, Diego León

1997. Compilación histórica de la Universidad de Guanajuato. Guanajuato, México: Centro de Investigaciones Humanísticas, Universidad de Guanajuato, Grupo Financiero Serfín, Guanajuato.

Román Alarcón, Rigoberto Arturo y Gustavo Aguilar

2010. "La reconversión del tejido productivo en Mazatlán: de la minería y el comercio a la pesca y el turismo, 1910-1970”. En Meyibó, Nueva Época, número 2, Universidad Autónoma de Baja California: pp. 57-93.

Romero Macías, Emilio y Antonio Santiago Cumbreras

2010. "Poblados y explotaciones mineras como fuentes de recursos del turismo rural: el Andévalo Occidental, Huelva (España). En Pasos. Revista de Turismo y Patrimonio Cultural, vol. 8, no. 4, España: pp. 583-594.

Sánchez Rangel, Oscar

2012. La transformación de la economía tradicional mexicana. Guanajuato: mutaciones costosas durante la primera mitad del siglo XX, México: El Colegio de México, Tesis de Doctorado en Historia.

Sánchez Rangel, Oscar

2015. "Inversión extranjera y minería. La reactivación de la producción de plata en el Guanajuato porfiriano". En María Luna Argudín y María José Rhi Sausi (coords.), Repensar el siglo XIX. Miradas historiográficas desde el siglo XX (pp. 188-215), Colección Biblioteca Mexicana. México: Fondo de Cultura Económica, Consejo Nacional para la Cultura y las Artes, Universidad Autónoma Metropolitana.

Saragoza, Alex

2001. "The selling of Mexico: tourism and the state, 1929-1952". En Gilbert Joseph, Anne Rubenstein y Eric Zolov (eds.), Fragments of a Golden age. The politics of culture in Mexico since 1940 (pp. 91-115). United States of America: Duke University Press.

Secretaría de Turismo del Gobierno del Estado de Guanajuato

2012. Compendio de la actividad turística. Estado de Guanajuato, México: Gobierno del Estado de Guanajuato.

Urry, John

1990. The tourist gaze: Leisure and travel in contemporary societies, Londres: Sage.

Valenzuela Rubio, Manuel, Antonio Palacios García y Carmen Hidalgo Giralt

2008. "La valoración turística del patrimonio minero en entornos rurales desfavorecidos. Actores y experiencias". En Cuadernos de Turismo, no. 22, Universidad de Murcia, España: pp. 231-260.

Vidaurri Arechiga, José Eduardo

2014. "Escorzo de la actividad turística en Guanajuato 1890-2010. Hacia una caracterización del turismo en la región. Primera Parte". En Colmena Universitaria, número 93, Universidad de Guanajuato: pp. 47-61. 


\section{Notas}

1 Agradezco a los dictaminadores anónimos, cuyos comentarios fueron muy útiles para la culminación del artículo. Este trabajo forma parte del proyecto colectivo registrado en la División de Ciencias Sociales y Humanidades de la Universidad de Guanajuato titulado "Espacios rurales y urbanos en Guanajuato durante la era liberal, 1900 -1929".

2 "Se filmará una película en Guanajuato", GDB, 1 de septiembre de 1944.

3 "La película "Bugambilia" grandioso éxito de la cinematografía nacional", GDB, 16 de octubre de 1945.

EN, 8 de diciembre de 1929 .

5 AHGEG, SG-TD, Memorándum del gobierno de Guanajuato para Ramón Beteta, secretario de Hacienda y Crédito Público, 1948.

6 Jorge Martín, "Guanajuato de Hogaño", EG, 7 de mayo de 1949; "Gobierno y Universidad abordan el problema de la industrialización de la vieja ciudad minera", GDB, 22 de junio de 1945.

7 AHUG, CPA, caja 20, exp. 46, Iniciativa para la formación del Comité Pro-Guanajuato, México, D.F., 16 de diciembre de 1922, Talleres de H. Barrales Sucr.

8 AHUG-CPA, Caja 26, Exp: Minas. Correspondencia profesional, 1900-1920, carta de Francisco Antúnez Echagaray a Ponciano Aguilar, 9 de diciembre de 1917.

9 POEG, 3 de enero de 1929, Artículo $5^{\circ}$ de la Ley sobre protección a la industria en el estado de Guanajuato, 21 de diciembre de 1928.

10 AHGEG, SG-TD, Industrias, Informe elaborado por Francisco Antúnez Echagaray para el gobernador de Guanajuato sobre las gestiones para promover el desarrollo del estado, México, D.F., 1 de marzo de 1929.

11 "La industria de la cerámica puede robustecer la economía de la capital del estado", GDB, 20 de mayo de 1945; "Gobierno y Universidad abordan el problema de la industrialización de la vieja ciudad minera", GDB, 22 de junio de 1945.

12 "Nueva industria en esta capital", EN, 12 de julio de 1925.

13 "Se aprobó el presupuesto para erigir el Palacio Federal", EG, 14 de octubre de 1944; "Está por terminarse la edificación de un palacio", GDB, 9 de diciembre de 1945.

14 AHGEG, SG-TD: Generalidades, Memorándum del gobierno de Guanajuato para Ramón Beteta, secretario de Hacienda y Crédito Público, 1948.

15 "La construcción del camino a Dolores Hidalgo pronto será un hecho", EN, 15 de julio de 1928.

16 "El Resurgimiento de Guanajuato pronto será un hecho. La construcción del Camino a Dolores reportará grandes beneficios en la vida económica del estado.", EN, 3 de noviembre de 1929; "Un llamado a todos los guanajuatenses. La Construcción de vías de comunicación traerá el resurgimiento de Guanajuato.”, EN, 10 de noviembre de 1929; AHGEG, SG-TD, Hacienda del estado, Informe del director de caminos del Estado al Gobernador en relación con la construcción de caminos del $1^{\circ}$ de septiembre de 1928 al 31 de diciembre de 1929”, Silao, Gto., 17 de marzo de 1930.

17 AHGEG, SG-TD, Generalidades de Fomento, carta de Francisco Antúnez y Estanislao Barrón, del Comité Local Pro-Turismo, al Departamento de Turismo de El Banco de México S.A., 10 de octubre de 1930.

18 "Excursionistas americanos", EN, 31 de enero de 1926; "Próximamente visitarán esta capital un gran número de excursionistas", EN, 3 de noviembre de 1929.

19 AHGEG, SG-TD, REF, Acuerdo del Ejecutivo de Guanajuato, 5 de septiembre de 1929.

20 AHGEG, Boletines, suscripciones, publicaciones y folletos, 3.93.

21 "Visita de una famosa escritora norteamericana", EN, 11 de marzo de 1934.

22 AHGEG, SG-TD, Turismo, carta de Carlos Mares Martínez a Mauro Visoso, Oficial Mayor del Gobierno de Guanajuato, México, D.F., 8 de junio de 1939.

23 POEG, 4 de enero de 1931, Ley sobre planeación del Estado de Guanajuato; AHGEG, SG-TD, Leyes, carta de Francisco Antúnez Echagaray a Rafael Rangel, Secretario General de gobierno de Guanajuato, Guanajuato, Gto., 21 de noviembre de 1930.

24 POEG, Decreto 88, H. XXXVIII Legislatura Constitucional del Estado de Guanajuato, Ley sobre protección y conservación de la ciudad de Guanajuato, declarándola población histórica y típica, mayo de 1942.

25 Carta de Francisco Antúnez Echagaray, presidente de la Comisión Pro-Turismo de la ciudad de Guanajuato, a los miembros de la Comisión, Guanajuato, Gto., 14 de octubre de 1929; "Atinadas consideraciones nos presenta la cámara de comercio" EG, 1949

26 "Honda indignación por el descuido de monumentos artísticos", EN, 23 de agosto de 1936. "El rector de la Universidad Nacional", GDB, 9 de abril de 1944.

27 "Sugestiones a Turismo", EG, 7 de mayo de 1949.

28 "Se habla de modernizar Guanajuato", GDB, 10 de octubre de 1941; "Guanajuato, un lugar turístico", GDB, 14 de octubre de 1941; "Nueva avenida en Guanajuato", GDB, 16 de diciembre de 1942; "La ciudad en la ruta turística" y "Afluirá el turismo a la ciudad. Se construyen y acondicionan hoteles para recibir excursionistas", EG, 10 de noviembre de 1945.

29 "Cómo puede subsanarse la escasez del agua”, EN, 14 de junio de 1925; “¿Faltará en Guanajuato el agua?, EN, 16 de agosto de 1925. "El agua de la Presa de Esperanza causa de enfermedades serias", EN, 28 de septiembre de 1930. "Vecinos que se quejan del pésimo servicio de aguas que hay en la ciudad", EN, 23 de agosto de 1936. "Guanajuato se envenena paulatinamente con el agua de uso diario", EG, 23 de abril de 1949.

Recibido: 